\title{
Epinephrine Secretion Induction
}

National Cancer Institute

\section{Source}

National Cancer Institute. Epinephrine Secretion Induction. NCI Thesaurus. Code C41509.

Epinephrine Secretion Induction consists of the initiation of activities involved in release across the cell membrane into the bloodstream from the adrenal medulla of a sympathomimetic hormone (epinephrine) that stimulates the alpha- and beta- adrenergic systems, causes systemic vasoconstriction and gastrointestinal relaxation, stimulates the heart, and dilates bronchi and cerebral vessels. Alteration of positive or negative feedback mechanisms can affect hormone release. 